\title{
Full developmental potential of mammalian preimplantation embryos is maintained after imaging using a spinning-disk confocal microscope
}

\author{
Pablo Juan Ross, Gloria Ines Perez, Tak Ko, Myung Sik Yoo, and Jose Bernardo Cibelli
}

BioTechniques 41:741-750 (December 2006)

doi 10.2144/000112310

\begin{abstract}
Fluorescent live imaging of cells and embryos at subcellular resolution poses significant challenges for biologists due to morbidity and mortality ensuing from phototoxicity. Here we report the use of a spinning-disk confocal microscope to image mouse and bovine preimplantation embryos without impairing their developmental potential. We also present data indicating that this imaging technique does not affect the functionality of subcellular components as assessed by reactive oxygen species (ROS) production, caspase activity, and DNA integrity. Spinning-disk confocal microscopy was also useful in determining cell number and allocation in transgenic bovine blastocysts. We conclude that this imaging method is suitable for monitoring preimplantation embryos.
\end{abstract}

\section{INTRODUCTION}

Fluorescent probes are being used extensively in developmental biology for various imaging techniques, including nontoxic live staining and visualization of expressed fluorescent proteins. These techniques have enabled the identification and tracing of numerous cellular and subcellular structures and molecules. Nonetheless, a number of limitations are associated with the use of existing microscopy techniques. For instance, live cell fluorescence microscopy using laserscanning confocal microscopy (LSCM) induces embryo damage, as the cells are exposed to high-intensity light (1). A couple of microscopic techniques that are less toxic to cells lend themselves to exploitation. Two-photon fluorescence microscopy has proven less toxic for live samples and was used by Squirrell and colleagues to image hamster preimplantation embryos with no signs of developmental damage (1). However, despite its lower phototoxicity, the use of two-photon fluorescence microscopy has been hindered by its high cost and demand for technical expertise. In contrast, spinning-disk confocal microscopy is significantly more affordable and causes no discernable cellular damage and, as a consequence, is becoming the method of choice for dynamic imaging of living cells $(2,3)$.

The spinning-disk technology uses a Nipkow disk, which was invented in 1884 (4) and adapted for optical microscopy in 1968 (5). In essence, the spinning-disk apparatus uses the same principle as LSCM to obtain confocal images (i.e., the excitation light goes through a small pinhole and then the fluorescence emission passes back through a pinhole, thus eliminating out-of-focus light). The main difference of the spinning-disk systems is that they rely on several confocal pinholes mounted on a perforated disk that rotates at high speed, thus merging the fluorescence into one uniform two-dimensional image. As a result, photobleaching and damage to the living cells are minimal. This technology is particularly useful in biological studies that require a high rate of microscopic imaging (2). Applications of the technique include, among other things, monitoring cytokinesis regulation (6), microtubules dynamics (7), $\mathrm{Ca}^{2+}$ and cAMP signaling (8), calcium dynamics (9), membrane receptor internalization (10), and microvasculature physiology (11).

We have extended the use of the spinning-disk confocal technology to image live mammalian embryos (mouse and bovine) without compromising their developmental potential. We hypothesize that by using fluorescent markers during preimplantation development, it should be possible to capture high-resolution images that can later be correlated with the developmental competence of the embryos. Such a procedure could be useful for monitoring embryos of certain species, particularly under experimental conditions in which few embryos develop into normal individuals. For instance, only about $20 \%-30 \%$ of bovine 2 cell embryos produced by in vitro fertilization (IVF) reach the blastocyst stage, and barely half of these generate 


\section{Research Reports}

a pregnancy when transferred into a synchronized recipient (12). In contrast, approximately $70 \%$ of in vivo produced bovine embryos, when transferred to the uterus of a recipient cow, give rise to healthy calves. Likewise, a dramatically lower developmental efficiency is seen in somatic cell nuclear transfer (SCNT)-derived embryos, in which only $1 \%-10 \%$ of the embryos transferred into the uterus develop into healthy offspring (13). This implies that most of the in vitro produced embryos are destined to die; yet it remains very difficult to identify the phenotype of a developmentally competent embryo. Therefore, there is an imminent need for an affordable and reliable method to assess preimplantation embryos before they are transferred into surrogate mothers.

Similarly, despite the fact that SCNT has been successfully implemented in several mammalian species, attempts to improve its efficiency are hindered by the lack of reliable markers to indicate when the somatic nucleus transferred into the oocyte has undergone complete genome reprogramming and when the resulting embryo is able to generate a live offspring once transferred into the uterus of a surrogate mother. To date, the only reliable marker for success of complete reprogramming is the birth of a healthy offspring. This implies a long waiting period-at least 9 months in cattle- to determine the health status of the cloned offspring. It is therefore of utmost importance to find alternative markers for determining the developmental competence of a cloned preimplantation embryo. Each marker should be easily detectable by noninvasive methods and must be nontoxic to the embryo.

Among traits that have been suggested as potential markers for reprogramming are gene expression and cell number and their allocation to embryonic cell lineages at the blastocyst stage. Gene expression can also be monitored using fluorescent reporters driven by gene-specific promoters. Such an approach has been used in the mouse to identify cloned embryos with higher chances of generating embryonic stem cells (14). Cell number and their allocation are determined, so far, by techniques that require the destruction of the embryos (15) and are inherently limited in terms of correlating any trait with developmental potential. Therefore, we have devised a methodology based on confocal resolution to determine blastocyst cell number and their allocation without destroying the embryo. This method emanated from our hypothesis that the spinning-disk technology, coupled with standard fluorescent illumination, could provide the required spatial resolution without compromising the viability of the embryo.

Here, we present data on the use of spinning-disk confocal microscopy to image bovine and mouse preimplantation embryos without compromising their in vitro development and functionality. In addition, imaged mouse embryos gave rise to healthy offspring. Moreover, this imaging technique allowed us to determine cell number and allocation in live bovine blastocysts, thus facilitating the association of preimplantation phenotypes (e.g., cell number and their allocation) with developmental potential.

\section{MATERIALS AND METHODS}

\section{Chemicals}

All materials were obtained from Sigma Aldrich (St. Louis, MO, USA) unless otherwise specified.

\section{Animals and Embryos}

CD1 mouse 2-cell embryos were collected from superovulated females 40 to $45 \mathrm{~h}$ after human chorionic gonadotropin (hCG). The embryos were stained with $300 \mathrm{nM}$ Molecular Probes $^{\text {TM }}$ MitoTracker ${ }^{\circledR}$ Red CMXRos (Invitrogen, Carlsbad, CA, USA) for 15 min. Outside the incubator and during imaging, embryos were maintained in HEPES-buffered HECM medium [HH; $114 \mathrm{mM} \mathrm{NaCl}, 3.2 \mathrm{mM} \mathrm{KCl}, 2$ $\mathrm{mM} \mathrm{CaCl} 2,0.5 \mathrm{mM} \mathrm{MgCl} 2,0.1 \mathrm{mM}$ sodium pyruvate, $2 \mathrm{mM} \mathrm{NaHCO} 3,10$ $\mathrm{mM}$ HEPES, $17 \mathrm{mM}$ sodium lactate, $1 \times$ minimum essential medium (MEM) nonessential amino acids, $100 \mathrm{IU} / \mathrm{mL}$ penicillin $\mathrm{G}, 100 \mu \mathrm{g} / \mathrm{mL}$ streptomycin, $3 \mathrm{mg} / \mathrm{mL}$ bovine serum albumin (BSA)] (16). After imaging, embryos were cultured in potassium simplex optimized medium (KSOM; Specialty Media, Phillipsburg, NJ, USA) at $37^{\circ} \mathrm{C}$ and $5 \% \mathrm{CO}_{2}$. Blastocyst development was assessed on day 4 after fertilization, and embryos were surgically transferred into the uterus of day 2-3 pseudopregnant CD1 recipients. The pregnancies were allowed to proceed to term, and the number of pups born was recorded.

Bovine embryos were produced by in vitro fertilization of oocytes collected from slaughterhouse ovaries. The oocytes were matured in Medium 199 supplemented with $10 \%$ fetal bovine serum (FBS; HyClone, Logan, UT, USA), $1 \mu \mathrm{g} / \mathrm{mL}$ follicle-stimulating hormone (FSH; Sioux Biochemical, Sioux, IA, USA), $1 \mu \mathrm{g} / \mathrm{mL}$ luteinizing hormone (LH; Sioux Biochemical), 2.3 $\mathrm{mM}$ sodium pyruvate, and $25 \mu \mathrm{g} / \mathrm{mL}$ gentamicin sulfate (Gibco, Grand Island, NY, USA) and fertilized in vitro using Tyrode's albumin lactate pyruvate (TALP)-based medium (17). Embryo culture was performed in KSOM supplemented with $3 \mathrm{mg} / \mathrm{mL}$ BSA, but all manipulations outside the incubator were performed in $\mathrm{HH}$ medium. Eightto sixteen-cell embryos were collected for staining on day 3 after fertilization. After imaging, the embryos were cultured in KSOM supplemented with $3 \mathrm{mg} / \mathrm{mL} \mathrm{BSA}$ and $10 \% \mathrm{FBS}$ at $38.5^{\circ} \mathrm{C}$ and $5 \% \mathrm{CO}_{2}$.

All procedures involving animals were approved by the Michigan State University IACUC (East Lansing, Michigan, USA).

\section{Mitochondria Imaging}

In vitro generated bovine 8-cell embryos and in vivo produced mouse 2-cell embryos were stained with MitoTracker Red CMXRos (300 $\mathrm{nM}$ in $\mathrm{HH}$ medium for $15 \mathrm{~min}$ ) and individually imaged using a spinningdisk confocal module (CARV; Atto Bioscience, Rockville, MD, USA) attached to a Nikon TE2000-U microscope and a $40 \times$ Plan Fluor oil objective (1.3 NA; Nikon, Tokyo, Japan). The sample was excited with a $120 \mathrm{~W}$ metal halide lamp (X-Cite ${ }^{\circledR}$ 120, Fluorescence Illumination System; EXFO, Quebec, Montreal, Canada) at full intensity, using a fluorescent 


\section{Research Reports}

A
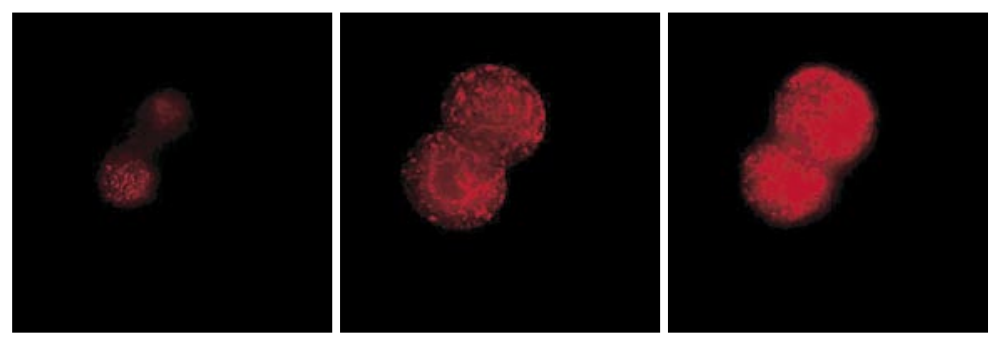

B
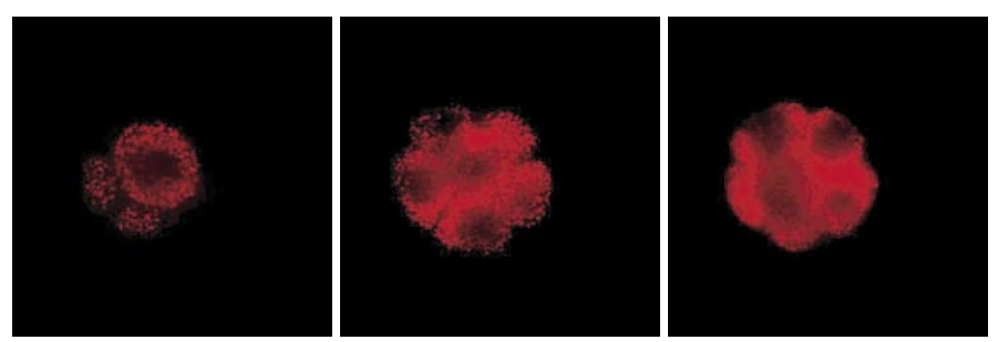

Figure 1. Embryos stained with MitoTracker Red and imaged using the CARV system. (A) Mouse 2-cell embryo and (B) bovine 8-cell embryo. Different $Z$ planes of the same embryo are shown.

filter set (exciter HQ575/50x, dichroic Q610LP, emitter HQ640/50m; Chroma Technology, Rockingham, VT, USA). Images were captured with an electron multiplying charge-coupled device (EMCCD) camera fitted with on-chip multiplication gain (Cascade 512B; Roper Scientific, Tucson, AZ, USA), using MetaMorph software (Universal Imaging, Downingtown, PA, USA). Sets of exposures of different durations were taken.

\section{Measurement of Reactive Oxygen Species}

Reactive oxygen species (ROS) formation was determined through the use of $2^{\prime}, 7^{\prime}$-dichlorodihydrofluorescein diacetate (DCHFDA; Molecular Probes, Invitrogen). The DCHFDA dye is membrane permeant; upon entering the cell, the acetate groups are hydrolyzed, creating a membrane impermeant form of the dye (DCHF). When present, endogenous reactive oxygen species will oxidize this polar form of the dye to a quantifiable fluorogenic compound (DCF); by proxy, this represents the level of ROS present in the cell, which can be detected by fluorescent microscopy with excitation and emission settings at 488 and $525 \mathrm{~nm}$, respectively. ROS content was evaluated before and after confocal imaging to determine the amount of ROS generated, and the average fluorescence intensity was measured using MetaMorph software. Embryos were pretreated with $10 \mathrm{nM}$ DCHFDA for $15 \mathrm{~min}$ prior to imaging. ROS production was calculated as the proportion of fluorescence intensity observed after imaging compared with the level registered just before imaging. To corroborate that the substrate was activating properly, a positive control was included that involved exposing the embryos to epifluorescence for 5$10 \mathrm{~s}$, which has been previously shown to increase ROS content in embryos (1).

\section{Caspase 3 Activity Assay}

Caspase 3 activity was measured using the cell-permeable fluorogenic substrate (PhiPhiLux-G1D2; OncoImmunin, Gaithersburg, MD, USA) according to the manufacturer's recommendations. Briefly, after imaging, the embryos were incubated in $10 \mu \mathrm{L} \quad 10 \mu \mathrm{M}$ PhiPhiLux-G1D2 substrate solution in RPMI 1640. After incubation for $1 \mathrm{~h}$ at $37^{\circ} \mathrm{C}$ in the dark, the embryos were rinsed once in $\mathrm{HH}$ medium and imaged using epifluorescence microscopy fitted with fluorescein isothiocyanate (FITC) fluorescence filters. Average fluorescence intensity was measured using MetaMorph software. As a positive control, embryos were incubated in 100 $\mu \mathrm{M} \mathrm{H}_{2} \mathrm{O}_{2}$ in $\mathrm{HH}$ medium for $1 \mathrm{~h}$.

\section{Pancaspase Activity Assay}

Active caspases were measured using the Caspatag TM Pan-Caspase In Situ Assay kit (Chemicon International, Temecula, CA, USA), following the manufacturer's specific protocol. This reagent is based on the use of a caspase inhibitor that is labeled with carboxyfluorescein (FAM-VAD-FMK). The inhibitor is cell permeable and noncytotoxic. Once inside the cell, it binds covalently to active caspases and becomes sequestered in the cytoplasm. Unbound reagent will diffuse out of the cell during the washing steps. The green fluorescent signal is a direct measure of the amount of active caspase(s) present in the cell at the time the reagent was added. After labeling for $1 \mathrm{~h}$, the embryos were imaged using epifluorescence microscopy fitted with FITC fluorescence filters. Average fluorescence intensity was measured using MetaMorph software. Positive (embryos incubated in $500 \mathrm{mM} \mathrm{H}_{2} \mathrm{O}_{2}$ for $1 \mathrm{~h}$ ) and negative controls (nonimaged embryos) were included in all assays.

\section{Comet Assay}

The comet assay was performed to determine the effect of confocal imaging using the CARV system on embryonic DNA integrity. DNA damage was analyzed in whole embryos. The CometAssay ${ }^{\mathrm{TM}}$ kit (Trevigen, Gaithersburg, MD, USA) was used according to the manufacturer's protocol. Briefly, the embryos were rinsed in phosphate-buffered saline (PBS) solution and placed on the supplied slides. Then, $75 \mu \mathrm{L}$ lowmelting-point agarose were added. The slide was left at $4^{\circ} \mathrm{C}$ for $30 \mathrm{~min}$ for the agarose to solidify. Next, the embryos were lysed in cold buffer and their DNA was denatured in alkaline solution ( $\mathrm{pH}>13.0)$. Electrophoresis was performed in neutral buffer (1X Tris-borate-EDTA, pH 8.3; TBE), and finally DNA was stained using $\mathrm{SYBR}{ }^{\circledR}$ Green. The comets were imaged using a standard epifluorescence microscope with the appropriate excitation and emission filters. The comet length and total intensity were determined using MetaMorph software. 


\section{Research Reports}

A group of treated embryos and a group of negative controls were loaded separately on each slide. As a positive control, embryos were treated with 500 $\mu \mathrm{M} \mathrm{H}_{2} \mathrm{O}_{2}$ in $\mathrm{HH}$ medium for $30 \mathrm{~min}$.

\section{Somatic Cell Nuclear Transfer}

Oocytes that had matured for 16-18 $\mathrm{h}$ were separated from the surrounding cumulus cells by vortex agitation in $\mathrm{HH}$ medium containing $1 \mathrm{mg} / \mathrm{mL}$ hyoluronidase for $5 \mathrm{~min}$. All micromanipulations were carried out in $\mathrm{HH}$ medium supplemented with $7.5 \mu \mathrm{g} / \mathrm{mL}$ cytochalasin B and 20\% FBS. Oocyte enucleation was performed, using a $15-\mu \mathrm{m}$ (internal diameter) glass pipet, by aspirating the metaphase II (MII) plate in a small volume of surrounding cytoplasm. Oocytes were previously stained in KSOM medium containing $5 \mu \mathrm{g} / \mathrm{mL}$ of bisbenzidine for $10 \mathrm{~min}$. Enucleation was performed under UV light to ensure the removal of the oocyte's DNA. Donor cells were dissociated by treatment with $10 \mathrm{IU} / \mathrm{mL}$ pronase in $\mathrm{HH}$ media for 5 min. A single cell was inserted into the perivitelline space of the enucleated oocyte using a $20-\mu \mathrm{m}$ (internal diameter) glass pipet. Bovine skin fibroblasts expressing nuclear-targeted HcRed 1 protein driven by chicken- $\beta$ actin promoter and cytomegalovirus (CMV) enhancer were used as donor cells. Oocyte-cell couplets were fused in sorbitol fusion medium ( $250 \mathrm{mM} \mathrm{D}$ sorbitol, $0.5 \mathrm{mM} \mathrm{MgOAc}$, and $1 \mathrm{mg} / \mathrm{mL}$ BSA). Oocytes were placed in a fusion chamber with a $0.5-\mathrm{mm}$ gap between electrodes, mechanically aligned, and fused with a single direct current pulse of $200 \mathrm{~V} / \mathrm{mm}$ for $15 \mu \mathrm{s}$.

Activation of the nuclear transfer (NT) units was performed 2-4 h after fusion by treatment with $5 \mu \mathrm{M}$ ionomicin (Calbiochem, San Diego, CA, USA) for $4 \mathrm{~min}$ followed by incubation in KSOM media containing $2 \mathrm{mM}$ dimethylaminopurine (DMAP) for $4 \mathrm{~h}$. After activation, the NT units were rinsed several times in $\mathrm{HH}$ medium and cultured in $400-\mu \mathrm{L}$ drops of KSOM media supplemented with $3 \mathrm{mg} / \mathrm{mL}$ BSA under mineral oil at $38.5^{\circ} \mathrm{C}$ and $5 \% \mathrm{CO}_{2}$ in air. On day 3 $(\mathrm{NT}=$ day 0$)$, the embryo culture drops were supplemented with 10\% FBS and cultured under the same conditions until day 7, when blastocysts were recovered for imaging.

\section{Plasmid Vector Construction}

We used a plasmid vector containing a chicken $\beta$-actin promoter and a CMV enhancer driving expression of nuclear localized HcRed 1 protein. The CMV promoter of pHcRed1-Nuc plasmid

Table 1. Embryonic Development After Confocal Fluorescence Imaging

\begin{tabular}{|c|c|c|c|c|c|c|}
\hline Experiment & Treatment & $\begin{array}{l}\text { Z Planes } \\
\text { (n) }\end{array}$ & $\begin{array}{c}\text { Exposure Time } \\
\text { (ms) }\end{array}$ & $n$ & $\begin{array}{l}\text { BI Ratea } \\
(\%)\end{array}$ & $\begin{array}{c}\text { Hatched BI Rate } \\
(\%)\end{array}$ \\
\hline \multirow[t]{2}{*}{ Bovine 1} & Control & - & - & 63 & 36 & 36 \\
\hline & $30-\mathrm{z}, 50 \mathrm{~ms}$ & 30 & 50 & 56 & 37 & 26 \\
\hline \multirow{2}{*}{ Bovine 2} & $60-\mathrm{z}, 100 \mathrm{~ms}$ & 60 & 100 & 68 & 32 & 21 \\
\hline & $90-\mathrm{z}, 100 \mathrm{~ms}$ & 90 & 100 & 68 & 37 & 19 \\
\hline \multirow[t]{2}{*}{ Bovine 3} & Stained & - & - & 55 & 25 & 22 \\
\hline & & & & & & Pups Bornc \\
\hline \multirow[t]{6}{*}{ Mouse } & Control & - & - & 87 & $79^{d}$ & $17 / 28(57 \%)$ \\
\hline & Stained & - & - & 106 & $91 \mathrm{e}$ & $22 / 36(61 \%)$ \\
\hline & $5-\mathrm{z}, 50 \mathrm{~ms}$ & 5 & 50 & 122 & $96 \mathrm{e}$ & $37 / 66(56 \%)$ \\
\hline & $10-\mathrm{z}, 50 \mathrm{~ms}$ & 10 & 50 & 55 & $93^{e}$ & 19/39 (49\%) \\
\hline & $20-z, 50 \mathrm{~ms}$ & 20 & 50 & 54 & $89 \mathrm{~d}, \mathrm{e}$ & $11 / 29(38 \%)$ \\
\hline & $30-z, 50 \mathrm{~ms}$ & 30 & 50 & 97 & $30^{f}$ & $0 / 27$ \\
\hline \multicolumn{7}{|c|}{$\begin{array}{l}\text { Mouse embryos were tested at the 2-cell stage, while bovine embryos were evaluated at the } 8 \text { - and } 16 \text {-cell stage. All embryos were stained with MitoTracke } \\
\text { Red CMXRos and imaged using the CARV system. Bl, blastocyst. } \\
\text { a,bPercentage of embryos developing to the indicated stage over embryos imaged. } \\
\text { cPups born/embryos transferred. Only pregnant recipients considered. } \\
\text { de,e,Groups with different superscripts were significantly different }(P<0.05) \text {. }\end{array}$} \\
\hline
\end{tabular}




\section{Research Reports}

A
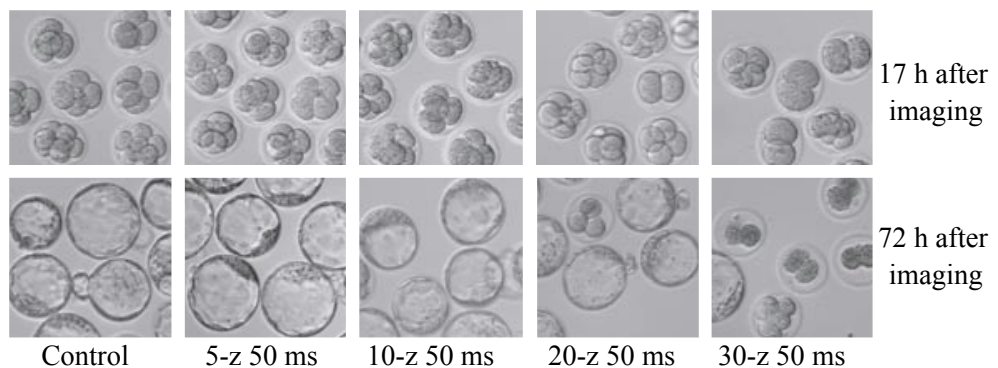

B


Figure 2. Effect of imaging mouse embryos using a spinning-disk confocal microscope. (A) Brightfield image of embryos at indicated times after imaging. (B) Bright-field and fluorescent images of embryos labeled with dichlorofluorescein (DCF) for determination of reactive oxygen species (ROS) production. (C) Quantitation of ROS production. Bars indicate mean fluorescent intensity of each embryo relative to the intensity before imaging. Positive control, exposure to $5 \mathrm{~s}$ of epifluorescence. (D) Mean comet length (open bar) and caspase activity (gray bar) of embryos after imaging using the CARV system. Positive control, incubation in $100 \mathrm{mM} \mathrm{H}_{2} \mathrm{O}_{2}$ for $1 \mathrm{~h}$. The total number of embryos evaluated per group is in parentheses above the bars. Different letters indicate statistical differences between treatments $(P<0.05)$.

(Clontech, Mountain View, CA, USA) was excised by digesting with AseI and Eco47III restriction enzymes and replaced with a multiple cloning site (MCS) from pBluescript ${ }^{\circledR}$ II KS+ plasmid (Stratagene, La Jolla, CA, USA), which was previously amplified by PCR using T3 and T7 primers.
Then, the chicken $\beta$-actin promoter and CMV IE enhancer combination (Sal1 to EcoR1) from the pCX-EGFP vector (18) was subcloned into the MCS upstream of HcRed1 protein sequence.

\section{Differential Staining for Inner Cell Mass and Trophectoderm Cell Number Determination of Bovine Blastocysts}

The zona pellucida of each blastocyst was removed by incubation in $0.5 \%$ pronase for $1 \mathrm{~min}$. Embryos were exposed to a 1:5 dilution of rabbit anti-pig whole serum for $1 \mathrm{~h}$, rinsed three times for 5 min each in $\mathrm{HH}$ media, and placed into a 1:5 dilution of guinea pig complement containing $10 \mu \mathrm{g} / \mathrm{mL}$ propidium iodide and 10 $\mu \mathrm{g} / \mathrm{mL}$ bisbenzimide for $1 \mathrm{~h}$. After a short rinse in $\mathrm{HH}$ media, embryos were mounted in $70 \%$ glycerol solution on a glass slide under a coverslip. Total cell count and allocation of cells to trophectoderm (TE) and inner cell mass (ICM) lineages were determined using the Nikon TE2000-U epifluorescence microscope. TE cells were observed as red nuclei and ICM cells as blue nuclei.

\section{Confocal Imaging for ICM and TE Cell Number Determination of Bovine Blastocysts}

Bovine blastocysts produced by IVF, parthenogenesis, and SCNT were imaged to determine their cell number and allocation using a spinning-disk confocal microscope followed by three-dimensional deconvolution. Imaging was performed in embryos stained with $5 \mu \mathrm{M}$ Syto 16 (Molecular Probes, Invitrogen), a cell-permeant nuclear stain, in HH medium for $15 \mathrm{~min}$ and in transgenic embryos expressing HcRed1 fluorescent protein targeted to the nucleus. Embryos were placed with the ICM facing the objective lens in between two coverslips separated from each other by $150 \mu \mathrm{m}$. The sample was excited with a $120 \mathrm{~W}$ metal halide lamp (X-Cite 120, Fluorescence Illumination System) using a fluorescent filter set specific for the fluorophores (Syto 16: exciter HQ470/40x, dichroic Q495LP, emitter HQ525/50m; HcRed: exciter HQ575/50x, dichroic Q610LP, emitter 


\section{Research Reports}

A

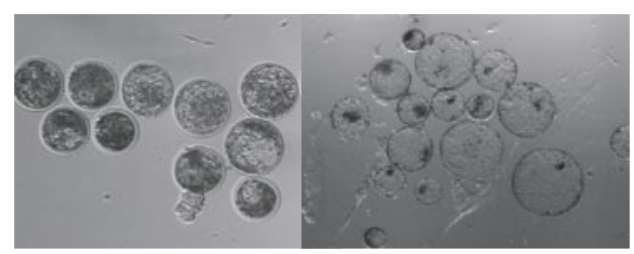

B
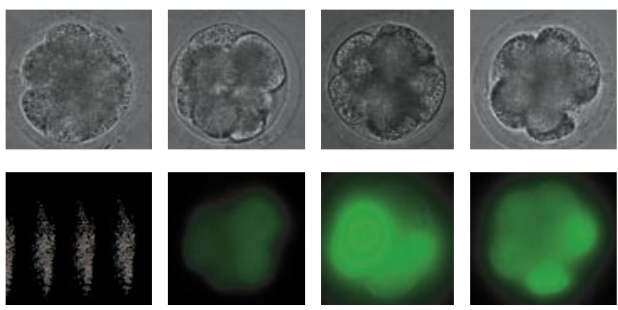

Negative Control

$60-\mathrm{z}$

$60-\mathrm{z}$

Positive

$50 \mathrm{~ms}$

Control

C
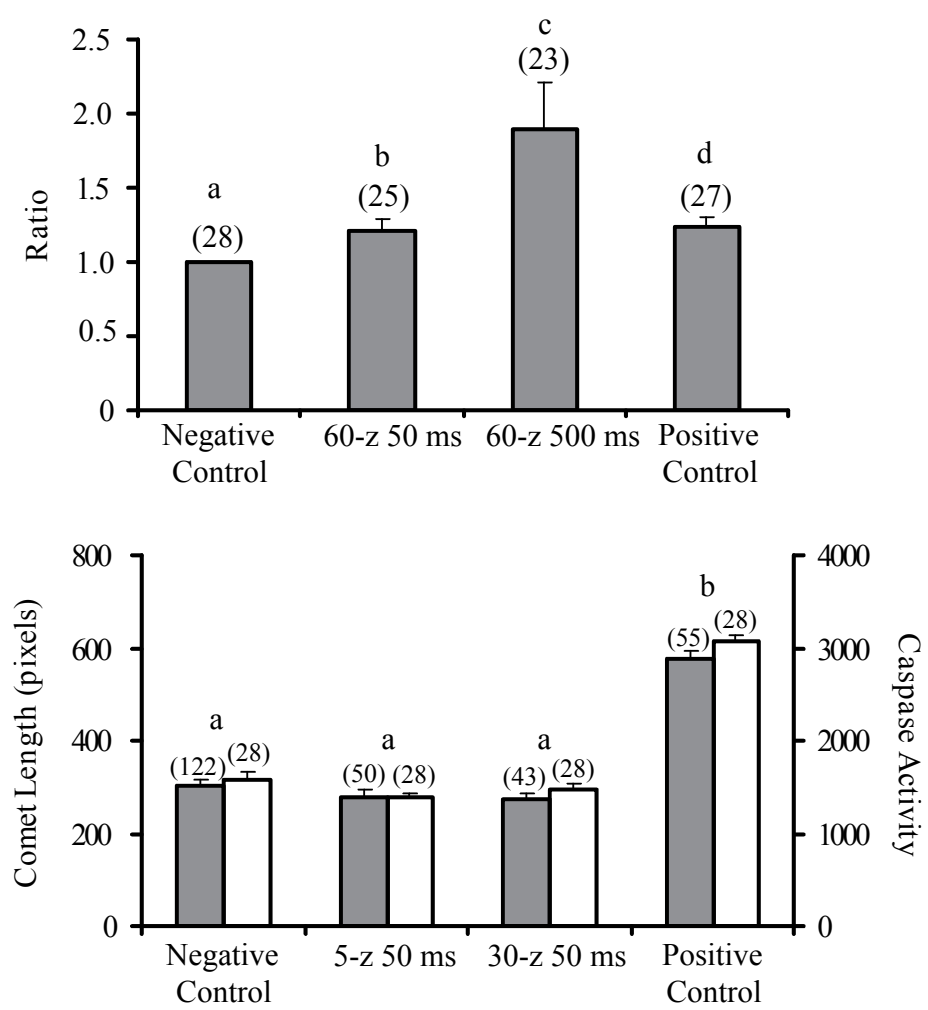

Figure 3. Effect of imaging bovine embryos using a spinning-disk confocal microscope. (A) Brightfield image of blastocysts and hatched blastocysts. (B) Bright-field and fluorescent images of embryos labeled with dichlorofluorescein (DCF) for determination of reactive oxygen species (ROS) production. (C) Quantitation of ROS production. Bars indicate mean fluorescent intensity of each embryo relative to the intensity before imaging. Positive control, exposure to $10 \mathrm{~s}$ of epifluorescence. (D) Mean comet length (open bar) and caspase activity (gray bar) of embryos after imaging using the CARV system. Positive control, incubation in $500 \mathrm{mM} \mathrm{H}_{2} \mathrm{O}_{2}$ for $1 \mathrm{~h}$. The total number of embryos evaluated per group is in parentheses above the bars. Different letters indicate statistical differences between treatments $(P<0.05)$.

HQ640/50m; Chroma Technology). A Z stack of the embryo was acquired every $5 \mu \mathrm{m}$ using a spinning-disk confocal system, and the images were processed using AutoQuant (Media
Cybernetics, Silver Spring, MD, USA) and MetaMorph software. All nuclei were marked by drawing a contour on the image for each focal plane and were then counted. Nuclei in the periphery of the embryo were assigned to the TE, with the remaining nuclei assigned to the ICM.

\section{Statistical Analysis}

Fluorescence intensity data and comet length were analyzed by analysis of variance (ANOVA), using the MIXED procedure of SAS (SAS Institute, Cary, NC, USA) considering different variances (i.e., heteroskedasticity). Multiple mean comparisons were performed using the Tukey adjustment. Means and standard error of means were used to plot the data. Embryo and pup development were analyzed by $\chi^{2}$ test.

\section{RESULTS}

\section{Viability of Preimplantation Embryos Following Confocal Imaging}

Following imaging using the CARV system (Figure 1), mouse 2-cell embryos did not differ in blastocyst rate formation from stained nonimaged controls when subjected to a series of 5,10 , or 20 fluorescence exposures taken along the z-axis of the embryo at $50-\mathrm{ms}$ exposures. Surprisingly, embryos stained with MitoTracker had a greater rate of development to blastocysts compared with nonstained embryos. Transferring these embryos to pseudopregnant females did not affect the proportion of pups born (Table 1). However, there was a tendency for the 20-z, 50-ms exposures to decrease pup rate $(P=0.085)$. All pups born reached adulthood and generated healthy offspring.

We found that a set of 20 exposures of $50 \mathrm{~ms}$ each is the maximum tolerated by mouse embryos. Beyond this threshold, blastocyst development decreased abruptly, and embryos were incapable of generating offspring (Table 1 and Figure 2A).

Bovine embryos imaged at the 8- to 16-cell stage showed no differences in blastocyst development $(\mathrm{d} 7.5$; day $0=$ IVF) or number of hatched blastocysts (d11) in comparison with nonimaged embryos (Table 1 and Figure 3A). Despite numerous attempts, we were 


\section{Research Reports}

unable to find a level of exposure that disturbed embryonic development in the bovine embryo.

ROS content was evaluated immediately before and after confocal imaging to determine the amount of ROS generated. Mouse embryos exposed to imaging significantly increased their ROS content when compared with nonimaged controls. When the embryos were subjected to 5 or 30 consecutive 50 -ms exposures, the increase in ROS content was $7 \%$ and $50 \%$, respectively, over the levels registered before imaging (Figure 2, B and C). Similarly, a significant increase was observed in bovine embryos after exposure (Figure 3 , B and C).

We also evaluated whether high exposure to fluorescent light induces caspase activation directly or following accumulation of ROS. The data revealed that pancaspases (Figures 2D and 3D) and caspase 3 (see Supplementary Figure S1 available online at www. BioTechniques.com) were not activated when measured $1.5 \mathrm{~h}$ after imaging.

Since DNA damage could also be induced by fluorescent light or by the accumulation of ROS in imaged embryos, we decided to evaluate DNA integrity using the comet assay $(19,20)$. When we evaluated bovine and murine embryos subjected to different exposure conditions, we did not find any differences in the length or intensities of DNA tails (Figure 2D, 3D, and Supplementary Figure S2) compared with nonexposed controls. DNA damage was not different among embryos exposed to different levels of imaging.

\section{Live Visualization and Quantification of Nuclei in Bovine Embryos}

Currently, differential staining is the only reliable method to differentiate cells that belong to the ICM from those that are TE $(15,21)$. Using our spinning-disk confocal microscope, we set out to compare the results obtained using the traditional differential staining with our live staining in the same embryo (Figure 4). We stained IVF and parthenogenetically activated day 7 bovine blastocysts using Syto



Figure 4. Comparison between live imaging and differential staining techniques for counting inner cell mass (ICM), trophectoderm (TE), and total cell numbers in bovine embryos.
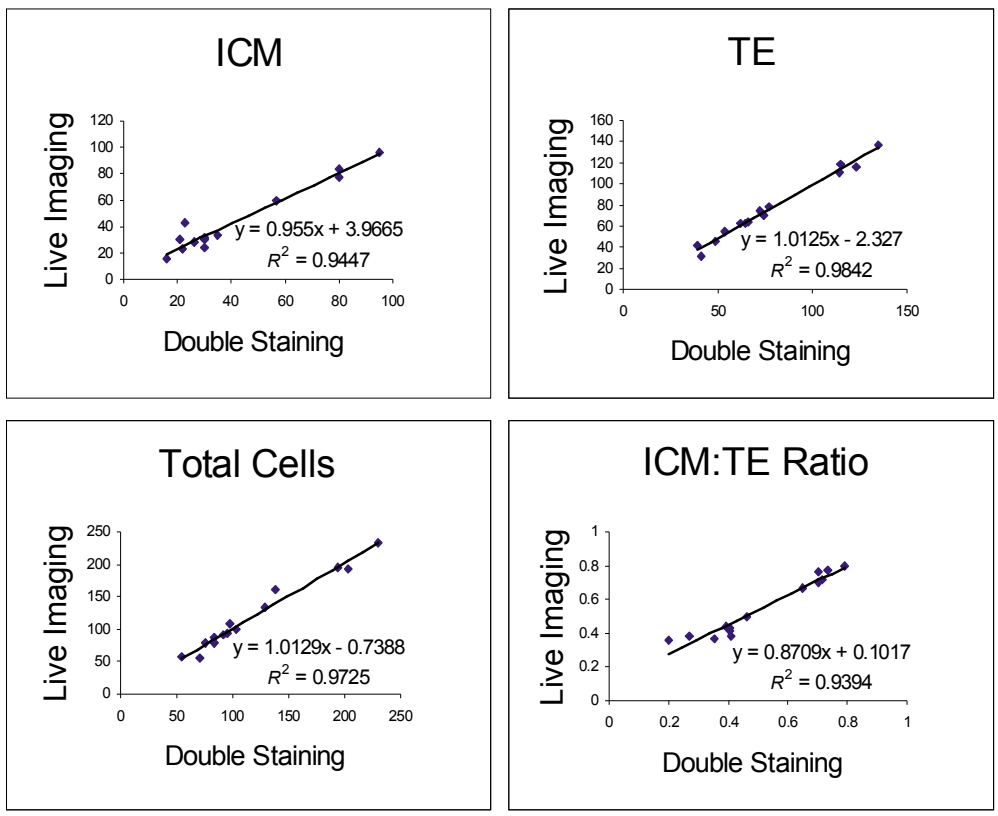

16. $\mathrm{Z}$ stack pictures were taken using a spinning-disk confocal microscope. This system produces a good spatial separation, both in $\mathrm{XY}$ and $\mathrm{Z}$ planes, allowing visualization of individual nuclei in the blastocyst-stage embryo. Complete $\mathrm{Z}$ series of optical sections at $5-\mu \mathrm{m}$ intervals were acquired from each embryo. The images stack was then subjected to three-dimensional deconvolution using AutoQuant software, and the nuclei were manually counted. Allocation of nuclei to ICM or TE was based on their position in the embryo. Nuclei located at the periphery of the embryo were considered TE, while the cells in the interior of the blastocyst were considered ICM. Subsequently we performed standard differential staining in the same embryo and correlated the results using both methods. Both techniques for cell counting and allocation agreed greatly. We observed a very high correlation and a slope no different than $1(P>0.1)$ for the number of ICM, TE, and total cells, and for the ICM:TE cell ratio with regression analysis (Figure 4). This data indicate that cell number and allocation can be precisely determined in bovine blastocysts using a live imaging system without destroying the embryo.

To determine the accuracy of our in vivo cell counting method, we stained HcRed-expressing embryos with Syto 16. Subsequently, these embryos were imaged as described, using fluorescent filters for HcRed and for Syto 16. The processed images were compared for each embryo. We found a high level of agreement between HcRed and Syto 16 images for blastocyst cell number and allocation (Figure 5). 


\section{Research Reports}

\section{DISCUSSION}

Microscopic imaging of live preimplantation embryos constitutes an excellent model for evaluating phototoxicity on cellular proliferation, differentiation, and morphogenesisprocesses that are all well delineated in embryos. Moreover, these changes can be easily assessed in a short period of time using simple visualization tools. In the present studies, we chose to use the spinning-disk confocal microscope to image bovine and mouse embryos at their most sensitive stage of development, the time of embryonic genome activation, which occurs at the 2-cell stage in mice and at the 8- to 16-cell stage in cattle. After this stage, surviving embryos undergo compaction at the morula stage and, later, cavitation and cell differentiation into the first two embryonic lineages (i.e., ICM and TE) to form a blastocyst. Comparison of the two models, side by side, allowed us to establish significant species differences in the sensitivity of embryos to fluorescent illumination.

In general, bovine preimplantation embryos showed an amazing adaptability to stress conditions (e.g., long exposures to fluorescent illumination), which probably reflects the presence and effectiveness of stress protective mechanisms. Although far more sensitive than the bovine embryo, below certain levels of exposure, mouse embryos developed and gave rise to live pups at approximately the same ratio as unexposed embryos. We therefore conclude that the spinningdisk confocal microscope is a suitable tool for imaging preimplantation embryos, provided that exposure is limited to a maximum of 20 exposures of $50 \mathrm{~ms}$ each.

It should be noted that in the present studies we utilized a spinningdisk confocal microscope that uses a Nipkow disk and standard fluorescent illumination; the spinning-disk confocal apparatus can be also equipped with laser illumination, but this light energy was not used in the present studies. Although we have not compared the two spinning-disk systems, we would expect a higher phototoxicity when imaging using a fluorescent source of higher energy (i.e., lasers). In our case, a mercury halide lamp was used; this source of illumination produces light in a similar spectrum to commonly used mercury arc lamps, but it has greater stability in both the short- and long-term and a much longer working lifespan. Our illumination source was used at $100 \%$ intensity; however, the level of illumination received at the sample was reduced to $7 \%$. This reduction is the result of passing the light through the spinning disk that, in this case, acts as a $93 \%$ neutral density (ND) filter.

Phototoxicity is not a well-understood phenomenon, although it occurs in most forms of fluorescence microscopy and has been previously associated with ROS production (22). Light-induced ROS production can occur as a consequence of fluorophores reacting with
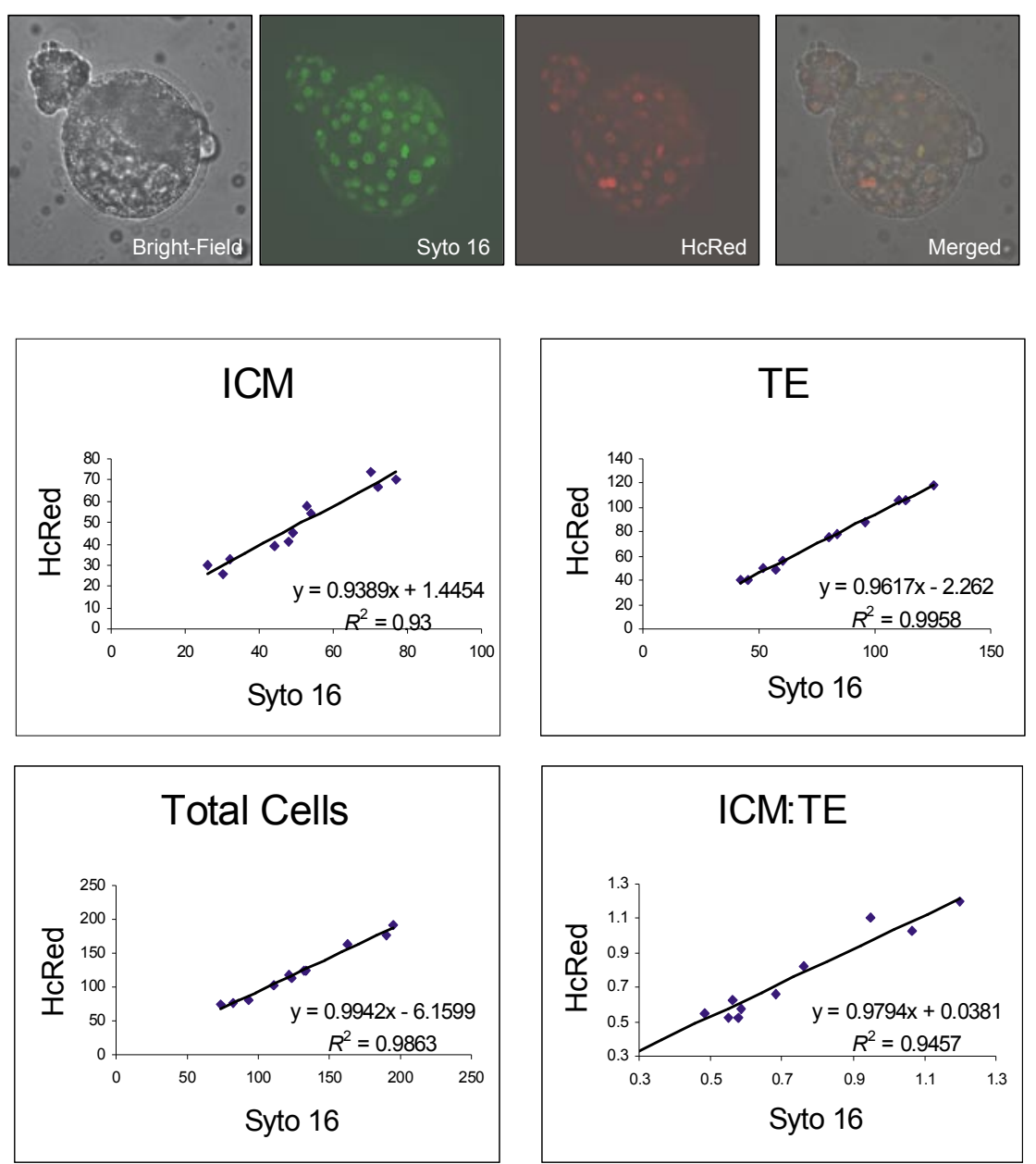

Figure 5. Comparison between HcRed and Syto 16 live imaging technique for counting inner cell mass (ICM), trophectoderm (TE), and total cells in transgenic somatic cell nuclear transfer (SCNT) embryos. oxygen or following light-induced damage in cellular components. It has been reported that blue light (450-490 nm) stimulates hydrogen peroxide $\left(\mathrm{H}_{2} \mathrm{O}_{2}\right)$ production in cultured cells by photoreduction of flavin, which in turn activates flavin-containing oxidases (23). Both bovine and mouse embryos exposed to imaging significantly increased their ROS content as compared with nonimaged controls. However, only mouse embryos were negatively affected by high levels of cytoplasmic ROS. We speculate that bovine embryos are probably enriched with high levels of cytoplasmic antioxidants and ROS scavengers, allowing them to quickly bring down high cytoplasmic levels of ROS, which otherwise could be detrimental for further embryo development. 
As the negative effects of imaging on embryo development can be the consequence of both the fluorescent illumination and the excitation of fluorochromes in the sample, we assessed the effect of fluorescent illumination on embryos stained with MitoTracker Red, a mitochondrial probe known to be nontoxic for embryonic development. Here, we also found surprising and significant differences between the two species. While MitoTracker did not have any effect on bovine embryo development, mouse embryos stained with MitoTracker had a greater rate of development to blastocysts. However, the reasons for this remains unknown to us at this time.

Activation of caspases and DNA damage are two major events in determining the fate of a cell toward apoptosis. However, in the present studies, we found no evidence to indicate that the imaging technique affected the functionality of either one of these subcellular components. Therefore, activation of caspases and DNA damage do not appear to be an immediate consequence of exposure to the spinning-disk confocal imaging. The possibility exists, however, that activation of caspases, DNA damage, and apoptosis occur at a later time point or that a mechanism independent of caspases could be activated.

As a possible application of preimplantation embryonic screening, we investigated the possibility of determining cell number and their allocation to embryonic cell lineages at the blastocyst stage in bovine embryos. This characteristic is usually considered as an indication of embryo quality, although no data are yet available to associate cell number and allocation directly with developmental potential of blastocysts. The lack of these kinds of data are a consequence of a technical limitation that could be resolved by the use of the spinning-disk confocal microscopy. Differential staining of ICM and TE cells in mammalian blastocysts can only be performed using differential staining techniques, which can be achieved with a variety of different strategies (15). However, all of these techniques require the destruction of the embryo and hence are incompatible with embryonic development. Our data indicate that it is possible to determine cell number and allocation in embryos expressing a fluorescent protein targeted to the nuclei. Cell number and allocation were determined by capturing 30 images of $150 \mathrm{~ms}$ each at different focal planes. This exposure level represents an exposure to fluorescent illumination well below (approximately 6-fold lower) the highest exposure level we tested, which resulted in no detrimental effect for in vitro bovine embryos stained with MitoTracker Red. Unfortunately, the transgenic approach of this methodology limits its application to NT-derived embryos. It would be desirable if a nontoxic, live nuclear staining were available-essentially making this technology amenable to nontransgenic embryos. To that end, we have tested some live nuclear staining alternatives (e.g., Syto 16 and Draq5), but found that the staining itself was highly toxic (data not shown).

To our knowledge, this is the first report describing the use of spinningdisk confocal fluorescence microscopy for monitoring mammalian embryos and their development to term. Our results indicate that it is possible to image preimplantation embryos without compromising their developmental potential and functionality. Compared to mouse embryos, bovine embryos showed a greater tolerance to imaging when in vitro development was assessed. It remains to be determined whether these embryos are still capable of implanting and developing into healthy offspring when transferred into recipient cows. The use of this technology in embryological studies will enable us to correlate early preimplantation events with subsequent development in the same specimen.

\section{ACKNOWLEDGMENTS}

We would like to thank Amy Iager, Zeki Beyhan, and Jessica Armstrong for help with oocyte collections, Ricardo Felmer for help with nuclear transfer, Kerrianne Cunniff for assistance with embryo transfers, and David Smith for technical assistance with the CARV system.

\section{COMPETING INTERESTS STATEMENT}

The authors declare no competing interests.

\section{REFERENCES}

1.Squirrell, J.M., D.L. Wokosin, J.G. White, and B.D. Bavister. 1999. Long-term twophoton fluorescence imaging of mammalian embryos without compromising viability. Nat. Biotechnol. 17:763-767.

2. Wang, E., C.M. Babbey, and K.W. Dunn. 2005. Performance comparison between the high-speed Yokogawa spinning disc confocal system and single-point scanning confocal systems. J. Microsc. 218:148-159.

3. Nakano, A. 2002. Spinning-disk confocal microscopy - a cutting-edge tool for imaging of membrane traffic. Cell Struct. Funct. 27:349-355.

4. Nipkow, P. 1884. Elektrisches teleskop (electric telescope). Berlin, Germany. Kaiserliches patentamt, patent no. 30, 105.

5.Petran, M., M. Hadravsky, M.D. Egger, and R. Galambos. 1968. Tandem-scanning reflected-light microscope. J. Opt. Soc. Am. 58:661-664.

6. Bement, W.M., H.A. Benink, and G. von Dassow. 2005. A microtubule-dependent zone of active RhoA during cleavage plane specification. J. Cell Biol. 170:91-101.

7. Moore, A.T., K.E. Rankin, G. von Dassow, L. Peris, M. Wagenbach, Y. Ovechkina, A. Andrieux, D. Job, and L. Wordeman. 2005. MCAK associates with the tips of polymerizing microtubules. J. Cell Biol. 169:391-397.

8. Landa, L.R., Jr., M. Harbeck, K. Kaihara, O. Chepurny, K. Kitiphongspattana, O. Graf, V.O. Nikolaev, M.J. Lohse, et al. 2005. Interplay of $\mathrm{Ca} 2+$ and cAMP signaling in the insulin-secreting MIN6 \{beta\}-cell line. J. Biol. Chem. 280:31294-31302.

9. Hara, M., V. Bindokas, J.P. Lopez, K. Kaihara, L.R. Landa, Jr., M. Harbeck, and M.W. Roe. 2004. Imaging endoplasmic reticulum calcium with a fluorescent biosensor in transgenic mice. Am. J. Physiol. Cell Physiol. 287:C932-C938.

10. Galliera, E., V.R. Jala, J.O. Trent, R. Bonecchi, P. Signorelli, R.J. Lefkowitz, A. Mantovani, M. Locati, and B. Haribabu. 2004. Beta-arrestin-dependent constitutive internalization of the human chemokine decoy receptor D6. J. Biol. Chem. 279:2559025597.

11. Marrelli, S.P., M.S. Eckmann, and M.S. Hunte. 2003. Role of endothelial intermediate conductance $\mathrm{KCa}$ channels in cerebral EDHF-mediated dilations. Am. J. Physiol. Heart Circ. Physiol. 285:H1590-H1599.

12. Faber, D.C., J.A. Molina, C.L. Ohlrichs, D.F. Vander Zwaag, and L.B. Ferre. 2003 Commercialization of animal biotechnology. Theriogenology 59:125-138.

13. Wilmut, I., N. Beaujean, P.A. de Sousa, A. Dinnyes, T.J. King, L.A. Paterson, D.N. Wells, and L.E. Young. 2002. Somatic cell nuclear transfer. Nature 419:583-587. 


\section{Research Reports}

14. Boiani, M., S. Eckardt, H.R. Scholer, and K.J. McLaughlin. 2002. Oct4 distribution and level in mouse clones: consequences for pluripotency. Genes Dev. 16:1209-1219.

15. Van Soom, A., G. Vanroose, and A. de Kruif. 2001. Blastocyst evaluation by means of differential staining: a practical approach. Reprod. Domest. Anim. 36:29-35.

16. Seshagiri, P. and B. Bavister. 1989. Phosphate is required for inhibition by glucose of development of hamster 8-cell embryos in vitro. Biol. Reprod. 40:607-614.

17.Parrish, J.J., J.L. Susko-Parrish, M.L. Leibfried-Rutledge, E.S. Critser, W.H. Eyestone, and N.L. First. 1986. Bovine in vitro fertilization with frozen-thawed semen. Theriogenology 25:591-600.

18. Niwa, H., K.-i. Yamamura, and J.-i. Miyazaki. 1991. Efficient selection for highexpression transfectants with a novel eukaryotic vector. Gene 108:193-199.

19.Singh, N.P., M.T. McCoy, R.R. Tice, and E.L. Schneider. 1988. A simple technique for quantitation of low levels of DNA damage in individual cells. Exp. Cell Res. 175:184-191.

20. Morita, Y., G.I. Perez, F. Paris, S.R. Miranda, D. Ehleiter, A. HaimovitzFriedman, Z. Fuks, Z.H. Xie, et al. 2000. Oocyte apoptosis is suppressed by disruption of the acid sphingomyelinase gene or by sphingosine-1-phosphate therapy. Nat. Med. 6:1109-1114.

21. Van Soom, A., M. Boerjan, M.-T. Ysebaert, and A. de Kruif. 1996. Cell allocation to the inner cell mass and the trophectoderm in bovine embryos cultured in two different media. Mol. Reprod. Dev. 45:171-182.

22.Jou, M.J., S.B. Jou, M.J. Guo, H.Y. Wu, and T.I. Peng. 2004. Mitochondrial reactive oxygen species generation and calcium increase induced by visible light in astrocytes. Ann. N.Y. Acad. Sci. 1011:45-56.

23. Hockberger, P.E., T.A. Skimina, V.E. Centonze, C. Lavin, S. Chu, S. Dadras, J.K. Reddy, and J.G. White. 1999. Activation of flavin-containing oxidases underlies lightinduced production of $\mathrm{H}_{2} \mathrm{O}_{2}$ in mammalian cells. Proc. Natl. Acad. Sci. USA 96:62556260.

Received 10 April 2006; accepted 25 September 2006.

Address correspondence to Jose Bernardo Cibelli, B270 Anthony Hall, East Lansing, MI 48824-1225, USA. e-mail: cibelli@msu.edu

To purchase reprints of this article, contact: Reprints@BioTechniques.com 